\title{
Oxytocin-Augmented Social Cognitive Skills Training in Schizophrenia
}

\author{
Michael C Davis ${ }^{1,2}$, Michael F Green ${ }^{1,2}$, Junghee Lee ${ }^{1,2}$, William P Horan ${ }^{1,2}$, Damla Senturk ${ }^{3}$, \\ Angelika D Clarke ${ }^{1,2}$ and Stephen R Marder*,1,2 \\ 'Department of Psychiatry and Biobehavioral Sciences, UCLA Semel Institute for Neuroscience and Human Behavior, Los Angeles, CA, USA; \\ ${ }^{2}$ VA Desert Pacific Mental IIIness Research, Education, and Clinical Center, Los Angeles, CA, USA; ${ }^{3}$ Department of Biostatistics, UCLA Fielding \\ School of Public Health, Los Angeles, CA, USA
}

\begin{abstract}
Impairments in social cognition are common in schizophrenia and predict poor functional outcome. The purpose of this proof-of-concept randomized, parallel group clinical trial was to assess whether intranasal oxytocin $(\mathrm{OT})$, given before social cognitive training, enhances learning of social cognitive skills. Twenty seven male outpatients with schizophrenia participated in a 6-week (12 session) training on social cognitive skills. Training focused on three domains: facial affect recognition, social perception, and empathy. Subjects were randomly assigned (double blind) to receive either intranasal OTor placebo $30 \mathrm{~min}$ before each session. Participants did not receive OT between sessions or on the day of assessments. We evaluated scores on social-cognition measures, as well as clinical symptoms and neurocognition, at baseline, I week following the final training session, and I month later. Our prespecified primary outcome measure was a social-cognition composite score comprised of five individual measures. There were main effects of time (indicating improvement across the combined-treatment groups) on the social-cognition composite score at both I week and I month following completion of training. Subjects receiving OT demonstrated significantly greater improvements in empathic accuracy than those receiving placebo at both posttreatment and I month follow up. There were no OT-related effects for the other social cognitive tests, clinical symptoms, or neurocognition. This study provides initial support for the idea that OT enhances the effectiveness of training when administered shortly before social cognitive training sessions. The effects were most pronounced on empathic accuracy, a high-level social cognitive process that is not easily improved in current social cognitive remediation programs.
\end{abstract}

Neuropsychopharmacology (2014) 39, 2070-2077; doi:I0.1038/npp.20I4.68; published online 9 April 2014

\section{INTRODUCTION}

Social-cognition-or the cognitive processes that underlie our social interactions-is commonly impaired in individuals with schizophrenia (Penn et al, 1997). Among the processes that are often affected are emotion processing, social perception, theory of mind/mental state attribution, and attributional style/bias (Pinkham et al, 2013). These impairments are important because they are associated with poorfunctional outcome (Horan et al, 2012). As a result, recent attention has focused on treatment strategies for improving social-cognition.

A recent meta-analysis of 19 studies that examined social cognitive training programs in schizophrenia and related disorders found moderate to large effects of training on facial affect recognition (a function of emotion processing), small to moderate effects on theory of mind, and

*Correspondence: Dr SR Marder, VA Desert Pacific Mental Illness Research, Education, and Clinical Center, II30I Wilshire Boulevard, Building 210A, Los Angeles, CA 90073, USA, Tel: +310 2683647 , Fax: +310 268 4056, E-mail: marder@ucla.edu

Received II November 2013; revised 20 February 20।4; accepted 13 March 20।4; accepted article preview online 18 March 2014 insignificant effects on social perception and attributional style (Kurtz and Richardson, 2012). While training for improved-facial affect recognition has been repeatedly found effective (Statucka and Walder, 2013), the results from training other social cognitive functions have been less consistent. For example,we found that a program of SocialCognition Skills Training (SCST) was effective for improving facial-affect perception and emotion management but less effective for improving performance on more complex domains such as the theory of mind and attributional bias (Horan et al, 2011b). Considering that the effects of training interventions are variable-both across subjects and across social cognitive domains-we wondered whether the efficacy could be improved with a pharmacological augmentation strategy.

A number of factors led us to select oxytocin (OT) as a candidate compound for facilitating social cognitive training.OT is a peptide that acts as a mediator of pro-social behavior (Insel and Fernald, 2004). There is evidence that OT increases the salience of social information (Averbeck, 2010; Prehn et al, 2013); increases the eye gaze toward eyes on a face (Guastella et al, 2008); selectively improves empathic accuracy in individuals who are less socially proficient (Bartz et al, 2010); and improves clinical 
symptoms in schizophrenia (Feifel et al, 2010; Lee et al, 2013; Modabbernia et al, 2013). OT has also been reported to improve certain social cognitive processes in schizophrenia (Averbeck et al, 2011; Fischer-Shofty et al, 2013; Goldman et al, 2011; Pedersen et al, 2011). Accordingly, we recently found that single doses of OT led to improvement in higher order inferential processes (Davis et al, 2013), the socialcognition dimension that generally responds less consistently to training than lower-level cue detection (Horan et al, 2009).

Our interest in OT in this current study was not on its direct effects on social cognition, which we did not assess. Rather, we were interested in whether its pro-social effects and its effects on the salience of social information would enhance the effectiveness of a psychosocial intervention, SCST. Because our goal was to evaluate the effects of OT on training, it was important to distinguish any direct effects of OT on social-cognition measures $v s$ effects on learning. Hence, we administered OT only before each SCST session. Our evaluations of social cognition, neurocognition, and clinical psychopathology were administered at least a week after patients received their final dose of OT. As a result, improvements in social cognition and other measures could only be related to learning (augmented by OT) and not to direct drug effects on the outcome measures. In this proof-ofconcept study, we used a shortened version of SCST (12 sessions over 6 weeks) that addressed three social cognitive processes (empathy, social perception, and facial affect recognition). We assessed outcome measures for each of these three processes, in addition to measures of other social cognitive process not specifically trained, at baseline, 1 week after completion of training, and at a 4-week follow up.

\section{MATERIALS AND METHODS}

\section{Participants}

Subjects included 27 male outpatients who met DSM-IV criteria for schizophrenia, as confirmed by the StructuredClinical Interview for DSM-IV. All of the subjects were clinically stable on an antipsychotic medication, with no dose change $>10 \%$ within 3 months of study entry. Exclusion criteria included inability to perform informed consent, history of hyponatremia, or history of epilepsy or traumaticbrain injury. Subjects were recruited from VA, UCLA, and community outpatient mental health clinics. After complete description of the study to the subjects, written-informed consent was obtained. All procedures were in accordance with the ethical standards of the Institutional Review Boards at both UCLA and VA Greater Los Angeles.

\section{Study Design}

One week before receiving study drug and beginning training, subjects received a performance battery of socialcognition tasks (described below), the MATRICS Consensus Cognitive Battery (MCCB) (Nuechterlein et al, 2008), and clinical symptom assessments with the Brief PsychiatricRating Scale (BPRS) (Overall and Gorham, 1962) and the Clinical-Assessment Interview for Negative Symptoms (CAINS) (Kring et al, 2013). One week later, subjects began SCST training in groups that included individuals receiving
OT or placebo. Subjects were randomized to intranasal OT (40 IU) or a matched placebo within each training group. Double-blind drug was administered $30 \mathrm{~min}$ before the SCST session and not on any other days. The social-cognition measures and the MCCB were re-administered at 1 week and 1 month after the final SCST session. The BPRS and the CAINS were re-administered at 1 week posttreatment. Adverse events were monitored by a side effect checklist and the Columbia Suicide Severity Rating Scale (C-SSRS) (Posner et al, 2011) which were administered at baseline and at 1 week and 1 month posttreatment.

\section{Pharmacological Treatment}

OT nasal spray (50 IU/ml) and an otherwise-identical placebo nasal spray were compounded by Inland Compounding Pharmacy (Loma Linda, CA, USA). Nasal sprays were prepared in $30-\mathrm{ml}$ multi-use bottles, calibrated to dispense $0.1 \mathrm{ml}$ per puff. Subjects were instructed to spray four puffs into each nostril, for a total dose of 40 IU OT (or equivalent volume of placebo spray). This dose was selected based on previous studies (Davis et al, 2013; Feifel et al, 2010).

\section{Social Cognitive Skills Training}

All of the study subjects received SCST that was similar to the program described in our previous work (Horan et al, 2011a). The program was modified to include only 12 sessions, administered twice a week for 6 weeks. The training was administered in small group format (6-8 participants per group), and because randomization was within training groups, all groups included individuals assigned to both placebo and OT. Training sessions were conducted by two group leaders who were trained and supervised by the developer of the intervention. Four of the sessions focused on interpreting facial expressions and situation (facial affect recognition); four focused on interpreting nonverbal gestures and vocal cues (social perception); and the final four focused on improving empathic accuracy. Each session consisted of $10 \mathrm{~min}$ of review, $30 \mathrm{~min}$ of training with new material, and $20 \mathrm{~min}$ of practice exercises. The training utilized skill-building techniques that are commonly used in psychiatric rehabilitation. These include breaking down complex social cognitive processes into their components and automating these skills through repetition and practice.

\section{Social Cognition Measures}

We included three social cognition measures that covered abilities that were a focus of training in the SCST. In addition, we included two social cognitive measures that were used to assess generalization to social cognitive domains not specifically covered in the training.

Measures assessing trained domains. These social-cognition measures included: facial affect recognition: participants were asked to identify facial expressions of basic emotions (happy, sad, angry, afraid, surprised, disgusted, or neutral) in still digital images from the standardized stimulus set developed by Ekman (Ekman and Friesen, 1976). Profile of Nonverbal Sensitivity (PONS): 
the PONS (Rosenthal et al, 1979) is used to assess social perception. Scenes of this videotape-based measure last $2 \mathrm{~s}$ and contain facial expressions, voice intonations, and/or bodily gestures of a Caucasian female. The empathic accuracy task: in this task (Lee et al, 2011), participants watch 12 (six positive and six negative) video clips, each lasting for 2.0-2.5 min. Each clip shows an individual (referred to as a 'target') while he/she discusses a positive or negative autobiographical event. For each clip, participants use a 9-point scale to rate how positive or negative they believe the target is feeling. The primary dependent measure is the correlation between participant ratings of the targets' emotions and the targets' ratings of their own emotions, calculated in 2-s time epochs throughout the clip. The mean correlation across clips provides an 'empathic accuracy' score for each participant.

Additional social cognitive measures. Managing emotions component of Mayer-Salovey-Caruso emotional intelligence test (MSCEIT). The MSCEIT (Mayer et al, 2003) assesses four components (branches) of emotional processing. This study focused on the Managing Emotions component, which has two subscales that examine the regulation of emotions in oneself and in one's relationships with others. The Awareness of Social Inference Test (TASIT): The TASIT (McDonald et al, 2003) is a videotape-based test of theory of mind in various social contexts. We used only part three, which assesses the ability to correctly identify sarcasm or lies.

\section{Statistical Analysis}

$T$-tests and Fisher's exact tests were used for baseline comparisons of age, sex, education level, marital status, and race between treatment groups. Generalized linear-mixed models (GLMM) with main effects of treatment (placebo and OT) and time (baseline, end of treatment, and 1-month follow up), attendance (number of completed training sessions), treatment by time interactions, and subject level random intercepts were used to model the longitudinal trajectories of the outcomes, employing an identity link (using SAS PROC MIXED). GLMM account for correlations between repeated measures within subjects and automatically handle missing data, producing unbiased estimates as long as observations are missing at random. Hence, all available observations from each subject were utilized in modeling via the GLMM. In addition to comparing scores on individual tests, we computed composite scores using the mean of $Z$-scores of individual measures (which were calculated using entire cohort means and SDs at baseline). These scores were MCCB nonsocial composite (consisting of all individual MCCB components except for MSCEITManaging Emotions) and social-cognition composite (consisting of PONS, TASIT, facial affect recognition, empathic accuracy, and MSCEIT-Managing Emotions). The prespecified primary outcome measure of this study was the social-cognition composite score. Estimates of effect size (Cohen's $d$ ) were computed using the difference between the OT and placebo mean changes from baseline to follow up assessments and the pooled (OT + placebo) SD of the change from baseline to follow up assessment.

\section{RESULTS}

See Figure 1 for a CONSORT diagram summarizing study screening, recruitment, randomization, and attrition. Thirteen patients were randomly assigned to the OT condition and 14 to placebo. There were no significant demographic differences between the patients assigned to active drug or

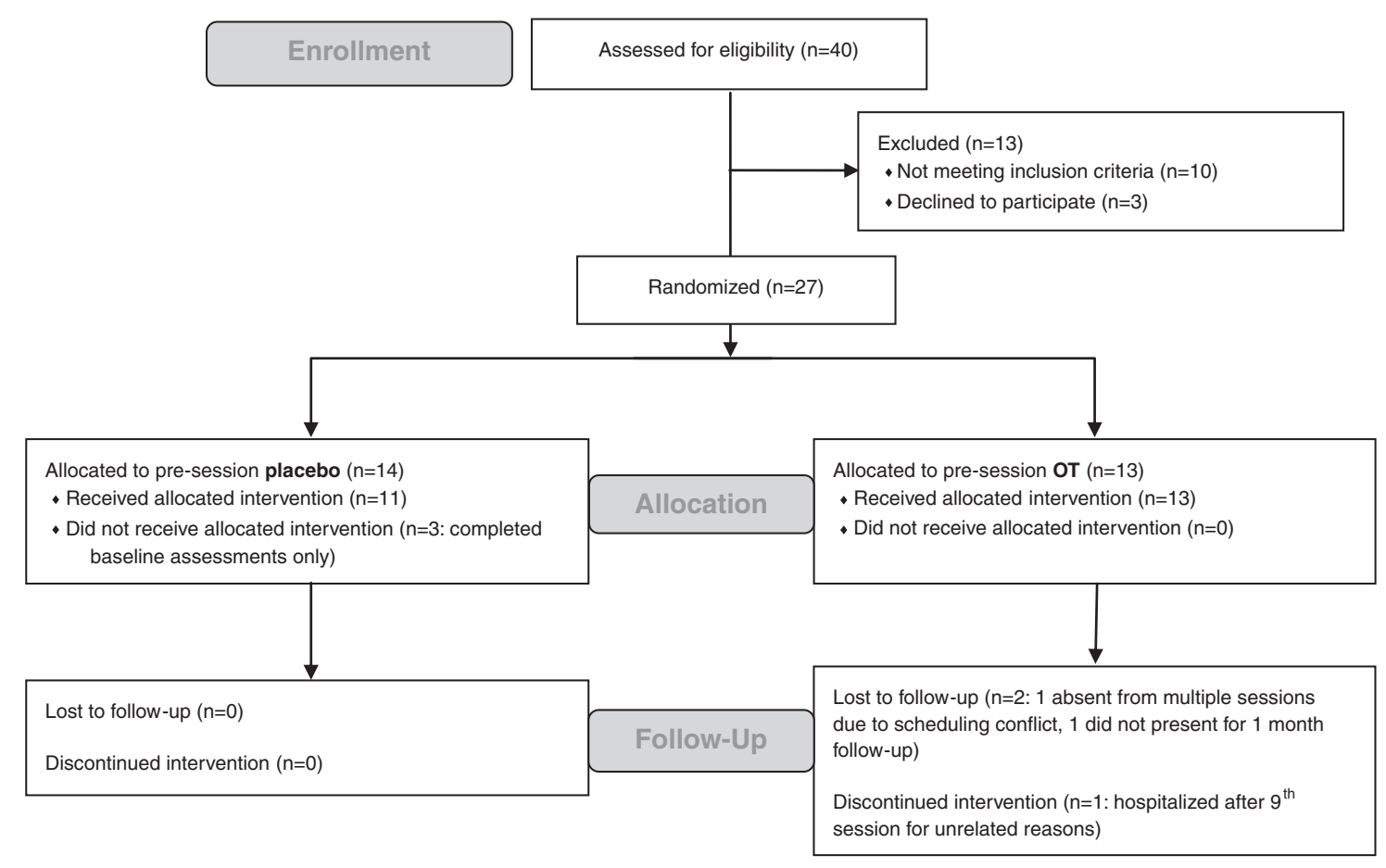

Figure I CONSORT diagram of participants in pharmacological approach to improve the outcome of social-cognition training. 
Table I Demographic Characteristics

\begin{tabular}{lcc}
\hline & Placebo $(\boldsymbol{n}=\mathbf{I 4})$ & Oxytocin $(\boldsymbol{n}=\mathbf{I 3})$ \\
\hline Age & $37.0(10.8)$ & $42.8(9.1)$ \\
Sex (male) & 14 & 13 \\
Personal education (years) & $12.0(0.8)$ & $12.5(1.7)$ \\
Parental education (years) & $12.6(3.4)$ & $14.8(2.2)$ \\
\% Never married & $86 \%$ & $85 \%$ \\
\% Nonwhite & $57 \%$ & $77 \%$ \\
\hline
\end{tabular}

Numbers represent mean values, with SDs in parentheses. For sex, numbers represent number of subjects. There were no statistically significant differences in demographic characteristics between the treatment groups.

placebo (Table 1). The number of subjects taking ant cholinergic medications (eg, benztropine, trihexyphenidyl, or diphenhydramine) was similar (3/14 in the placebo group and 2/13 in the OT group). There was no significant difference in group session attendance between groups (mean 8.6 sessions $\pm 4.7 \mathrm{SD}$ in the placebo group and mean 9.6 sessions $\pm 3.4 \mathrm{SD}$ in the OT group).

\section{Social Cognition}

(see Table 2): On our prespecified primary outcome measure, a social-cognition composite score, we found significant main effects of time, indicating improvement across the combined-treatment groups $(p=0.03$ at 1 week posttreatment and $p<0.01$ at 1 month). We also found significant main effects of time on individual measures of facial affect recognition $(p<0.0001$ at 1 week and 1 month posttreatment); the MSCEIT Managing Emotions total score ( $p=0.01$ at 1 week posttreatment and $p=0.03$ at 1 month); and the PONS total score ( $p=0.04$ at 1 month posttreatment). On our measure of empathic accuracy we found that subjects assigned to OT demonstrated significantly greater improvements than placebo on the total posttreatment $(p=0.03, d=0.92)$ and at 1 month $(p=0.03, d=0.98)$ (Figure 2). There were no effects of OT on any other individual social-cognitive measures or the social-cognition composite score.

\section{Neurocognition}

(see Table 2): We did not find effects of OT on basic neurocognition as measured by the MCCB nonsocial composite score. There was a main effect of time (across the combinedtreatment groups) on the MCCB nonsocial composite score between baseline and 1 month follow up $(p<0.01)$, suggesting overall improvement that may have been related to SCST and/or practice effects.

\section{Clinical Symptoms}

Clinical symptoms as measured by the BPRS and the CAINS (for negative symptoms) did not change significantly from baseline to posttreatment or 1 month follow up for either the OT or the placebo-treated patients (Table 3).

\section{Adverse Events}

There was one serious adverse event that was determined to be unrelated to study participation. In this case, the participant (who had been randomized to OT) had a recurrence of methamphetamine use that resulted in a brief psychiatric hospitalization for psychosis. He recovered fully and returned to the study groups. The only adverse events among study participants were occasional complaints about nasal irritation from the spray. These were equally distributed between OT and placebo patients. There were no changes in suicidal ideation or behaviors in either group, as assessed with the C-SSRS.

\section{DISCUSSION}

Compared with placebo, intranasal OT administered $30 \mathrm{~min}$ before SCST led to a significantly greater improvement in empathic accuracy. We are not aware of other psychosocial or pharmacological interventions that have been found to improve the acquisition of empathy skills in individuals with schizophrenia. However, we did not find effects of OT on our other measures of neurocognition and social cognition. Empathic accuracy is a high-level social cognitive skill that involves activation of the mirror neuron system as well as brain regions involved in mental state attribution (Zaki et al, 2009). The improvement in empathic accuracy was seen at the end of the training and after 1 month following OT administration, indicating that the beneficial effects were maintained beyond the acute effects of drug.

We found effects of time (all indicating improvement across the combined-treatment groups) on Ekman facialaffect recognition, MSCEIT total, and the social-cognition composite score between baseline and both posttreatment assessments. The improvement in the social-cognition measures is consistent with prior reports (Horan et al, 2009, 2011b; Roberts and Penn, 2009) finding similar effects. We also found time effects on the nonsocial MCCB composite posttreatment, which is consistent with prior study (Horan et al, 2011b). Though we did not have a control psychosocial intervention in this study, the time effects are most likely owing the SCST intervention, given their consistency with earlier controlled studies. The lack of an effect of social-cognition training on higher level inferential measures of social cognition such as theory of mind is also consistent with studies from the same groups.

There are a number of plausible mechanisms that can explain OT's effects on the learning of empathic accuracy. If patients treated with OT were less distracted by psychotic thought processes, they could be more attentive participants in training. This would be supported by previous findings that regular OT administration can reduce psychotic symptoms (Feifel et al, 2010). However, we did not find effects of OT on the learning of lower level social-cognition skills such as social perception or emotional processing, which may have occurred if this finding was related to effects on psychosis. Moreover, our previous study did not find acute effects of OT on clinical symptoms of schizophrenia (Davis et al, 2013), so improved attention via decreased psychotic symptoms is unlikely to be the mechanism. It is also possible that OT's effects on learning could have resulted from nonspecific cognitive-enhancing effects. 
Table 2 Social and Nonsocial Cognitive Assessments

\begin{tabular}{|c|c|c|c|c|c|c|c|c|c|c|c|}
\hline & & \multicolumn{3}{|c|}{ Placebo } & \multicolumn{3}{|c|}{ Oxytocin } & \multicolumn{4}{|c|}{ Effects $p<0.05$} \\
\hline & & $\begin{array}{c}\text { Baseline } \\
\text { mean (SD) } \\
(n=14)\end{array}$ & $\begin{array}{l}\text { Posttreatment } \\
\text { mean (SD) } \\
(n=I I)\end{array}$ & $\begin{array}{l}\text { I month } \\
\text { follow up } \\
\text { mean (SD) } \\
(n=10)\end{array}$ & $\begin{array}{c}\text { Baseline } \\
\text { mean (SD) } \\
(n=13)\end{array}$ & $\begin{array}{l}\text { Posttreatment } \\
\text { mean (SD) } \\
(n=I I)\end{array}$ & $\begin{array}{l}\text { I month } \\
\text { follow up } \\
\text { mean (SD) } \\
(n=I I)\end{array}$ & $\begin{array}{c}\text { Time } \\
\text { (placebo) }\end{array}$ & $\begin{array}{c}\text { Time } \\
\text { (oxytocin) }\end{array}$ & $\begin{array}{c}\text { Time } \\
\text { (combined } \\
\text { groups) }\end{array}$ & Oxytocin \\
\hline PONS & Total (of I I0) & $81.3(4.4)$ & $83.1(7.1)$ & $86.4(6.7)$ & $79.3(7.2)$ & $79.2(8.6)$ & $81.6(5.6)$ & & & $\begin{array}{l}p=0.04 \\
\text { at I month }\end{array}$ & \\
\hline \multirow[t]{3}{*}{ TASIT } & Total (of 64) & $49.5(6.1)$ & $49.1(6.4)$ & $51.3(4.4)$ & 46.1 (6.2) & $46.5(6.5)$ & $47.4(7.1)$ & & & & \\
\hline & Lie (of 32) & $26.2(3.6)$ & $25.4(4.7)$ & 27.1 (3.6) & $23.6(3.7)$ & $23.4(4.4)$ & $24.3(4)$ & & & & \\
\hline & Sarcasm (of 32) & $23.2(4.8)$ & 23.7 (5.4) & $24.2(4.9)$ & $22.5(5.3)$ & $23.1(5.8)$ & $23.1(6.6)$ & & & & \\
\hline $\begin{array}{l}\text { Facial affect } \\
\text { recognition }\end{array}$ & Fraction correct & $0.75(0.1)$ & $0.9(0.1)$ & $0.91(0.1)$ & $0.78(0.1)$ & $0.86(0.1)$ & $0.84(0.1)$ & $\begin{array}{c}p<0.000 \mid \\
\text { posttreatment } \\
\text { and at I month }\end{array}$ & $\begin{array}{c}p=0.0003 \\
\text { posttreatment } \\
\text { and } p=0.01 \\
\text { at I month }\end{array}$ & $\begin{array}{c}p<0.000 \mid \\
\text { posttreatment } \\
\text { and at I month }\end{array}$ & \\
\hline Empathic accuracy & Total $(r)$ & $0.66(0.2)$ & $0.64(0.2)$ & $0.65(0.1)$ & $0.62(0.2)$ & $0.7 \mid(0.2)$ & $0.7(0.2)$ & & & & $\begin{array}{c}p=0.03 \\
\text { posttreatment } \\
\text { and at I month }\end{array}$ \\
\hline $\begin{array}{l}\text { MSCEIT managing } \\
\text { emotions }\end{array}$ & Score (of I50) & $82.2(14)$ & $91(17)$ & $89.5(11)$ & $83.6(15)$ & $86.2(18)$ & $87.8(15)$ & $\begin{array}{c}p=0.02 \\
\text { posttreatment }\end{array}$ & & $\begin{array}{l}p=0.0 \mathrm{I} \\
\text { posttreatment } \\
\text { and } p=0.03 \\
\text { at I month }\end{array}$ & \\
\hline $\begin{array}{l}\text { Social-cognition } \\
\text { composite }\end{array}$ & Z-score & $0.035(0.8)$ & $0.35(0.8)$ & $0.5 \mid(0.6)$ & $-0.13(0.8)$ & $0.11(0.7)$ & $0.29(0.7)$ & & $\begin{array}{c}p=0.05 \\
\text { posttreatment }\end{array}$ & $\begin{array}{c}p=0.03 \\
\text { posttreatment } \\
\text { and } p<0.01 \\
\text { at I month }\end{array}$ & \\
\hline $\begin{array}{l}\text { MCCB nonsocial } \\
\text { composite }\end{array}$ & Z-score & $-0.09(0.6)$ & $0.11(0.5)$ & $0.4 \mid(0.5)$ & $0.09(0.8)$ & $0.08(0.9)$ & $0.16(1)$ & $\begin{array}{l}p=0.004 \\
\text { at I month }\end{array}$ & & $\begin{array}{l}\quad p<0.01 \\
\text { at I month }\end{array}$ & \\
\hline
\end{tabular}

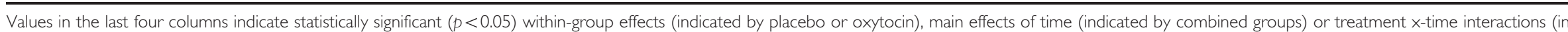
oxytocin column). 

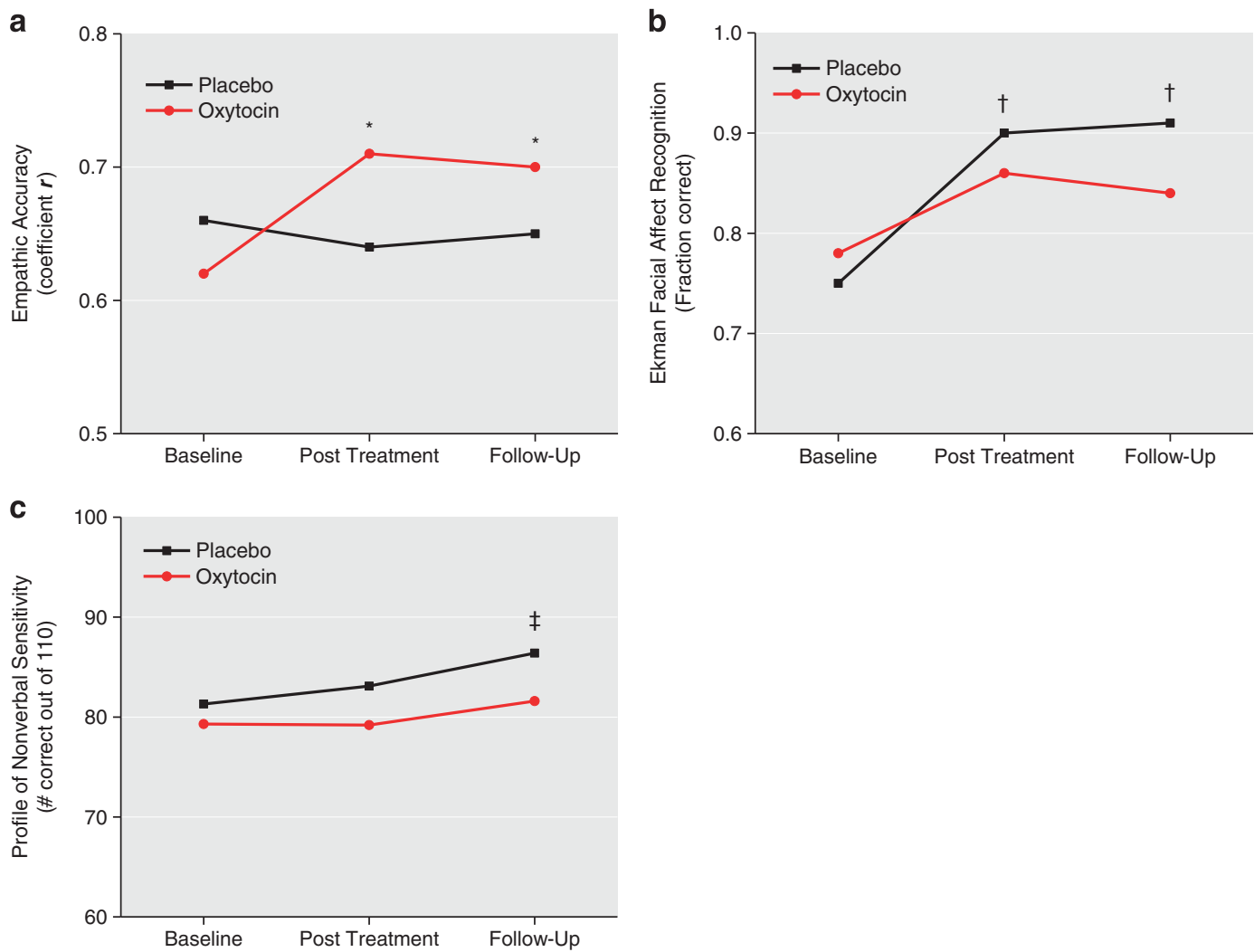

Figure 2 Social-cognitive performance on measures trained by SCST. (a) Empathic-accuracy correlation coefficients, (b) Ekman facial-affect recognition fraction correct, and (c) Profile of nonverbal sensitivity total scores for each treatment group at baseline, I week posttreatment, and I month follow up. ${ }^{*} p=0.03$ treatment $\times$ time interaction. ${ }^{\dagger} p<0.000$ I main effect of time. ${ }^{\ddagger} p=0.04$ main effect of time. All SDs can be found in Table 2.

Table 3 Clinical Symptom Assessments

\begin{tabular}{|c|c|c|c|c|c|c|c|c|c|}
\hline & & \multicolumn{4}{|c|}{ Placebo } & \multicolumn{4}{|c|}{ Oxytocin } \\
\hline & & $\begin{array}{l}\text { Baseline mean } \\
\quad(n=14)\end{array}$ & SD & $\begin{array}{l}\text { Posttreatment mean } \\
\qquad(n=I I)\end{array}$ & SD & $\begin{array}{l}\text { Baseline mean } \\
\quad(n=13)\end{array}$ & SD & $\begin{array}{l}\text { Posttreatment mean } \\
\qquad(n=I I)\end{array}$ & SD \\
\hline \multirow[t]{3}{*}{ CAINS } & Total & 19.1 & 6.9 & 17.8 & 7.1 & 22.0 & 10.2 & 21.1 & 11.6 \\
\hline & Experiential & 13.3 & 5.8 & 12.5 & 5.7 & 16.5 & 8.1 & 14.2 & 9.2 \\
\hline & Expressive & 5.7 & 3.1 & 5.2 & 3.4 & 5.4 & 4.7 & 6.8 & 4.5 \\
\hline
\end{tabular}

There were no statistically significant effects of time or treatment $x$ time interactions on any of the assessments below. Clinical assessments were not performed at the I month follow up visit.

OT has been reported to improve performance on subtests of the California Verbal Learning Test (Feifel et al, 2012), suggesting that chronic OT may improve verbal memory. Improvement in this basic cognition domain could have led to improved-content retention in the training setting. Again, the lack of effect of OT on lower level social cognitive skills does not support this as a mechanism for OT's effects.

There is evidence that OT can have effects on important social-cognitive functions which may have facilitated learning. This mechanism is consistent with our recent finding that single doses of OT acutely improve higher level social cognitive processes (Davis et al, 2013) as well as a study that found individuals with schizophrenia showed improvement in judging intimacy and kinship after receiving OT (Fischer-Shofty et al, 2013). It is thus plausible that OT facilitated the learning of social-cognition skills by increasing the salience of social information. Additional support for this mechanism includes evidence that men who were administered a single $24 \mathrm{IU}$ dose of OT recognized facial expressions at a lower intensity level than those administered placebo (Prehn et al, 2013), as well as the 
finding that OT enhanced pupil dilation in response to emotional faces, suggesting enhanced attention to social stimuli (Leknes et al, 2012). A hypothetical neural mechanism by which OT could increase the salience of social information is by modulating fast-spiking interneurons and thereby increasing the signal-to-noise ratio, as recently reported (Owen et al, 2013). It is not clear why OT only enhanced learning of empathic accuracy in this study and not facial affect recognition or social perception. This could be owing to either insufficient power to detect effects on these processes, or to OT having specific effects on a subset of neural circuits involved in social cognition.

We selected key outcome domains based on the modules that were covered in this 12 session version of SCST. The regular SCST includes 24 sessions and has a module on theory of mind (specifically on recognizing lie and sarcasm), but the abbreviated version used in this study did not. Empathic accuracy relies partially on cognitive empathy, which is similar to theory of mind (Zaki and Ochsner, 2011). However, theory of mind was not specifically trained in the empathy module.

It is important to note that the effects of $\mathrm{OT}$ on processing social information can vary depending on individual differences among subjects, including baseline abilities. For example, one study found that the performanceenhancing effects of OT were only seen in women with a lower interest in social interaction (Groppe et al, 2013). This is similar to the finding that OT's effects on empathic accuracy were only apparent in less socially-proficient individuals (Bartz et al, 2010) as well as that OT's effects on processing emotional faces were significantly greater in individuals with lower baseline emotional sensitivity (Leknes et al, 2012). It thus follows that OT may facilitate the learning of more complex social skills in individuals with schizophrenia who are limited in their ability to learn owing to difficulties in detecting subtle social signals.

One question that applies to this study, as well as other intranasal OT-treatment studies, is how long a given dose exerts its effects. While pharmacokinetic data regarding intranasal OT is limited, CSF levels of vasopressin (a nonapeptide similar in size and structure to OT) is elevated within $15 \mathrm{~min}$ of intranasal administration, with levels still increasing after $75 \mathrm{~min}$ (Born et al, 2002). Additionally, it was recently reported that intranasal administration of $24 \mathrm{IU}$ OT (a lower dose than that used in our study) resulted in elevated-plasma OT levels from 15-75 min and elevated-CSF levels at $75 \mathrm{~min}$ (with few time points sampled) (Stripes et al, 2013). Though elevated compartmental concentrations do not directly indicate receptor activity, it is reasonable to infer that intranasal OT was exerting a biological effect during the socialcognitive skills training sessions.

Important limitations of this study were the small sample size and our inability to control the possible effects of OT on clinical psychopathology and neurocognition. This was a preliminary study and we did not control for multiple comparisons. Hence, any conclusions from this study are necessarily tentative. Like any results from a preliminary study with multiple comparisons, our findings will await replication from larger samples. Important strengths include the double-blind design and the measurement of social cognition at least 1 week after OT administration. This later advantage suggests that effects of OT were owing to enhanced learning rather than OT's direct effects on social cognition. We also cannot prove that administering OT twice weekly before training did not cause a sustained increase in endogenous release. OT is known to have feed-forward regulatory properties, and long-term up regulation of endogenous OT systems by exogenous OT has been reported in rodents (Bowen et al, 2011).

Overall, this study demonstrated the feasibility and possible therapeutic benefit of administering OT before a psychosocial intervention targeting social cognition in individuals with schizophrenia. Improving empathic accuracy could help facilitate functional recovery in individuals with schizophrenia, given the significant associations between empathic abilities and social competence, functional capacity, and community functioning (Smith et al, 2013; Smith et al, 2012). These results support further investigation of this novel use of intranasal OT in schizophrenia as well as other psychiatric disorders associated with impaired socialcognitive function.

\section{FUNDING AND DISCLOSURE}

Dr Marder has received consulting fees from Abbott, Pfizer, Lundbeck, Bushranger Ingelheim, Bristol Meyers Squibb, Shire, Roche, Genentech, Otsuka, Targacept, and EnVivo. He has received grant supports from Amgen and Sunovion. Dr Green reports having been a consultant to Abbott Laboratories (AbbVie), Biogen, Dainippon Sumitomo Pharma, and Roche; he is a member of the scientific board for Mnemosyne; and he has received research funds from Amgen. This work was supported by the Hofmann Trust through the Brain and Behavior Research Foundation with an award to Dr Marder. The authors declare no conflict of interest.

\section{REFERENCES}

Averbeck BB (2010). Oxytocin and the salience of social cues. Proc Natl Acad Sci USA 107: 9033-9034.

Averbeck BB, Bobin T, Evans S, Shergill SS (2011). Emotion recognition and oxytocin in patients with schizophrenia. Psychol Med 42: 259-266.

Bartz JA, Zaki J, Bolger N, Hollander E, Ludwig NN, Kolevzon A et al (2010). Oxytocin selectively improves empathic accuracy. Psychol Sci 21: 1426-1428.

Born J, Lange T, Kern W, McGregor GP, Bickel U, Fehm HL (2002). Sniffing neuropeptides: a transnasal approach to the human brain. Nat Neurosci 5: 514-516.

Bowen MT, Carson DS, Spiro A, Arnold JC, McGregor IS (2011). Adolescent oxytocin exposure causes persistent reductions in anxiety and alcohol consumption and enhances sociability in rats. PLoS ONE 6: e27237.

Davis MC, Lee J, Horan WP, Clarke AD, McGee MR, Green MF et al (2013). Effects of single dose intranasal oxytocin on social cognition in schizophrenia. Schizophr Res 147: 393-397.

Ekman P, Friesen WV (1976). Pictures of Facial Affect. Consulting Psychologists Press: Palo Alto, CA.

Feifel D, Macdonald K, Cobb P, Minassian A (2012). Adjunctive intranasal oxytocin improves verbal memory in people with schizophrenia. Schizophr Res 139: 207-210. 
Feifel D, Macdonald K, Nguyen A, Cobb P, Warlan H, Galangue B et al (2010). Adjunctive intranasal oxytocin reduces symptoms in schizophrenia patients. Biol Psychiatry 68: 678-680.

Fischer-Shofty M, Brune M, Ebert A, Shefet D, Levkovitz Y, Shamay-Tsoory SG (2013). Improving social perception in schizophrenia: the role of oxytocin. Schizophr Res 146: 357-362.

Goldman MB, Gomes AM, Carter CS, Lee R (2011). Divergent effects of two different doses of intranasal oxytocin on facial affect discrimination in schizophrenic patients with and without polydipsia. Psychopharmacology 216: 101-110.

Groppe SE, Gossen A, Rademacher L, Hahn A, Westphal L, Grunder $G$ et al (2013). Oxytocin influences processing of socially relevant cues in the ventral tegmental area of the human brain. Biol Psychiatry 74: 172-179.

Guastella AJ, Mitchell PB, Dadds MR (2008). Oxytocin increases gaze to the eye region of human faces. Biol Psychiatry 63: 3-5.

Horan W, Kern R, Tripp C, Hellemann G, Wynn JK, Bell M et al (2011a). Efficacy and specificity of social cognitive skills training for outpatients with psychotic disorders. J Psychiatr Res 45: $1113-1122$.

Horan WP, Green MF, DeGroot M, Fiske A, Hellemann G, Kee K et al (2012). Social cognition in schizophrenia, Part 2: 12-month stability and prediction of functional outcome in first-episode patients. Schizophr Bull 38: 865-872.

Horan WP, Kern RS, Shokat-Fadai K, Sergi MJ, Wynn JK, Green MF (2009). Social cognitive skills training in schizophrenia: an initial efficacy study of stabilized outpatients. Schizophr Res 107: 47-54.

Horan WP, Kern RS, Tripp C, Hellemann G, Wynn JK, Bell M et al (2011b). Efficacy and specificity of social cognitive skills training for outpatients with psychotic disorders. J Psychiatr Res 45: $1113-1122$.

Insel TR, Fernald RD (2004). How the brain processes social information: searching for the social brain. Annu Rev Neurosci 27: 697-722.

Kring AM, Gur RE, Blanchard JJ, Horan WP, Reise SP (2013). The Clinical Assessment Interview for Negative Symptoms (CAINS): final development and validation. Am J Psychiatry 170: 165-172.

Kurtz MM, Richardson CL (2012). Social cognitive training for schizophrenia: a meta-analytic investigation of controlled research. Schizophr Bull 38: 1092-1104.

Lee J, Zaki J, Harvey P, Ochsner K, Green M (2011). Schizophrenia patients are impaired in empathic accuracy. Psychol Med 41: 2297.

Lee MR, Wehring HJ, McMahon RP, Linthicum J, Cascella N, Liu F et al (2013). Effects of adjunctive intranasal oxytocin on olfactory identification and clinical symptoms in schizophrenia: results from a randomized double blind placebo controlled pilot study. Schizophr Res 145: 110-115.

Leknes S, Wessberg J, Ellingsen DM, Chelnokova O, Olausson H, Laeng B (2012). Oxytocin enhances pupil dilation and sensitivity to 'hidden' emotional expressions. Soc Cogn Affect Neurosci 8: 741-749.

Mayer JD, Salovey P, Caruso DR, Sitarenios G (2003). Measuring emotional intelligence with the MSCEIT V2.0. Emotion 3: 97-105.

McDonald S, Flanagan S, Rollins J, Kinch J (2003). TASIT: a new clinical tool for assessing social perception after traumatic brain injury. J Head Trauma Rehabil 18: 219-238.
Modabbernia A, Rezaei F, Salehi B, Jafarinia M, Ashrafi M, Tabrizi $M$ et al (2013). Intranasal oxytocin as an adjunct to risperidone in patients with schizophrenia: an 8-week, randomized, doubleblind, placebo-controlled study. CNS Drugs 27: 57-65.

Nuechterlein KH, Green MF, Kern RS, Baade LE, Barch DM, Cohen JD et al (2008). The MATRICS Consensus Cognitive Battery, part 1: test selection, reliability, and validity. Am J Psychiatry 165: 203-213.

Overall JE, Gorham DR (1962). The brief psychiatric rating scale. Psychol Rep 10: 799-812.

Owen SF, Tuncdemir SN, Bader PL, Tirko NN, Fishell G, Tsien RW (2013). Oxytocin enhances hippocampal spike transmission by modulating fast-spiking interneurons. Nature 500: 458-462.

Pedersen CA, Gibson CM, Rau SW, Salimi K, Smedley KL, Casey $\mathrm{RL}$ et al (2011). Intranasal oxytocin reduces psychotic symptoms and improves Theory of Mind and social perception in schizophrenia. Schizophr Res 132: 50-53.

Penn DL, Corrigan PW, Bentall RP, Racenstein JM, Newman L (1997). Social cognition in schizophrenia. Psychol Bull 121: 114-132.

Pinkham AE, Penn DL, Green MF, Buck B, Healey K, Harvey PD (2013). The Social Cognition Psychometric Evaluation Study: Results of the Expert Survey and RAND Panel. Schizophr Bull, 31 May 2013 (Epub ahead of print).

Posner K, Brown GK, Stanley B, Brent DA, Yershova KV, Oquendo MA et al (2011). The Columbia-Suicide Severity Rating Scale: initial validity and internal consistency findings from three multisite studies with adolescents and adults. American Journal of Psychiatry 168: 1266-1277.

Prehn K, Kazzer P, Lischke A, Heinrichs M, Herpertz SC, Domes G (2013). Effects of intranasal oxytocin on pupil dilation indicate increased salience of socioaffective stimuli. Psychophysiology 50: 528-537.

Roberts DL, Penn DL (2009). Social cognition and interaction training (SCIT) for outpatients with schizophrenia: a preliminary study. Psychiatry Res 166: 141-147.

Rosenthal R, Hall JA, DiMatteo MR, Rogers PL, Archer D (1979). Sensitivity to nonverbal communication: the PONS test. Johns Hopkins University Press: Baltimore.

Smith MJ, Horan WP, Cobia DJ, Karpouzian TM, Fox JM, Reilly JL et al (2013). Performance-Based Empathy Mediates the Influence of Working Memory on Social Competence in Schizophrenia. Schizophr Bull, 31 December 2013 (Epub ahead of print).

Smith MJ, Horan WP, Karpouzian TM, Abram SV, Cobia DJ, Csernansky JG (2012). Self-reported empathy deficits are uniquely associated with poor functioning in schizophrenia. Schizophr Res 137: 196-202.

Statucka M, Walder DJ (2013). Efficacy of social cognition remediation programs targeting facial affect recognition deficits in schizophrenia: a review and consideration of high-risk samples and sex differences. Psychiatry Res 206: 125-139.

Striepens N, Kendrick KM, Hanking V, Landgraf R, Wullner U, Maier W et al (2013). Elevated cerebrospinal fluid and blood concentrations of oxytocin following its intranasal administration in humans. Scientific reports 3: 3440.

Zaki J, Ochsner K (2011). Reintegrating the study of accuracy into social cognition research. Psychol Inq 22: 159-182.

Zaki J, Weber J, Bolger N, Ochsner K (2009). The neural bases of empathic accuracy. Proc Natl Acad Sci USA 106: 11382-11387. 